\title{
Proton Pump Inhibitor Therapy in Eosinophilic Esophagitis: Current Role and Future Perspectives
}

Javier Molina-Infante, $M D^{1, *}$

Alfredo J. Lucendo, MD, PhD

\author{
Address \\ ${ }^{*}, 1$ Department of Gastroenterology, Hospital San Pedro de Alcantara, C/ Pablo \\ Naranjo s/n, 10003, Caceres, Spain \\ Email: xavi_molina@hotmail.com \\ ${ }^{2}$ Department of Gastroenterology, Hospital General de Tomelloso, Ciudad Real, \\ Spain
}

Published online: 12 March 2015

(C) Springer International Publishing AG 2015

This article is part of the Topical Collection on Food Allergy

Keywords Proton pump inhibitor-responsive esophageal eosinophilia - Eosinophilic esophagitis - Gastroesophageal reflux disease $\cdot$ Proton pump inhibitor

Abbreviations EDP Eosinophilic esophagitis diagnostic panel · EOE Eosinophilic esophagitis - GERD Gastroesophageal reflux disease - IL Interleukin - PPI Proton pump inhibitor - PPI-REE Proton pump inhibitor-responsive esophageal eosinophilia - STAT6 Signal transducer and activator of the transcription 6

\section{Opinion statement}

Eosinophilic esophagitis (EoE) is a chronic, immune/antigen-mediated esophageal disease characterized by symptoms related to esophageal dysfunction, eosinophilpredominant inflammation, and lack of response to high-dose proton pump inhibitor (PPI) therapy. Responsiveness to PPI therapy in patients with suspected EoE has been historically assumed as evidence of gastroesophageal reflux disease (GERD), but this concept has rapidly changed over the past few years. A novel phenotype, termed PPIresponsive esophageal eosinophilia (PPI-REE), was described in 2011. PPI-REE refers to patients who appear to have EoE, but who achieve complete remission after PPI therapy. Currently, a PPI trial is mandatory before diagnosing EoE since $30-40 \%$ of EoE patients will be eventually diagnosed with PPI-REE. Evolving evidence on PPI-REE suggests it is not simply GERD but actually a subphenotype of EoE, given the fact PPI-REE and EoE remain genetically and phenotypically indistinguishable. Instead of PPI-REE, the term PPIresponsive EoE might be more accurate to name this entity. PPI-REE might occur with either normal or pathological esophageal $\mathrm{pH}$ monitoring. PPI therapy can partially restore epithelial integrity and reverse allergic inflammation gene expression in PPI-REE. Whether 
esophageal barrier impairment is the cause of the effect of esophageal eosinophilia and whether PPI therapy primarily targets barrier or inflammation healing in PPI-REE have not been yet elucidated. The natural history of the disease, long-term doses, and duration of PPI therapy, as well as factors influencing symptom and/or inflammation relapse remain yet unknown. The mechanism as to why among identical patients, some respond to PPI therapy (PPI-REE) while others do not (EoE), warrants further research.

\section{Introduction}

Eosinophilic esophagitis (EoE) is a chronic, immune/ antigen-mediated esophageal disease characterized clinically by symptoms related to esophageal dysfunction and histologically by eosinophil-predominant inflammation. Currently, it represents the second most common cause of esophageal inflammation after gastroesophageal reflux disease (GERD) and the leading cause of dysphagia and food impaction in children and young adults [1]. The first Consensus Guidelines report on EoE was published in 2007 [2], proposing the following diagnostic criteria: (1) symptoms of esophageal dysfunction mainly dysphagia/food impaction, (2) esophageal eosinophilic infiltration (>15 eos/HPF), and (3) either absence of response to proton pump inhibitor (PPI) therapy or demonstration of normal esophageal acid exposure on $\mathrm{pH}$ monitoring. Therefore, it was suggested that either a response to PPI therapy or increased acid exposure on $\mathrm{pH}$ monitoring was consistent with GERD and, therefore, ruled out EoE. The premise underlying this recommendation was that GERD, as an acid-related disorder, was the only disease that could respond to the acid-suppressing ability of PPI treatment. An illustrative example of this thinking is given in the first case report of PPI-responsive esophageal eosinophilia (PPI-REE), published in 2006, in which two children and an adult with clinical, endoscopic, and histological data suggestive of EoE, achieved complete response to PPI therapy [3]. The authors literally concluded that "while these patients presentation was highly suggestive of allergic esophagitis, their symptoms, and the gross and histologic esophageal abnormalities normalized following treatment with a PPI, implicating acid reflux as the underlying cause."

\section{PPI-responsive esophageal eosinophilia}

\section{Initial description}

In 2011, the first prospective series was published, reporting that up to $50 \%$ of patients with an EoE phenotype (dysphagia/food impaction and $>15$ eos/HPF

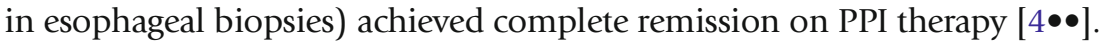
Clinical, endoscopic, and histological findings were indistinguishable between responders and nonresponders to PPI therapy. Of note, responsiveness to PPI therapy was observed not only in a majority of patients with GERD but also in $33 \%$ of those without evidence of GERD by endoscopy and $\mathrm{pH}$ monitoring.

The description of this new potential disease phenotype was acknowledged as one of the major breakthroughs in the updated guidelines for diagnosis and management of EoE published in 2011 [ $5 \bullet$. These guidelines identified an emerging body of literature and clinical experience describing a subset of EoE patients whose symptoms and histopathologic findings were responsive to PPI treatment and who might or might not have well-documented GERD. Additionally, several therapeutic/basic studies interestingly suggested a potential anti-inflammatory or barrier-healing role for PPI therapy in patients with suspected EoE [6]. 
In 2013, the first systematic review on PPI-REE [7 $\bullet$ ] highlighted PPI-REE as a prevalent entity ( $>30 \%$ of patients with suspected EoE) and its growing importance in literature, especially in adult patients. PPI-REE was significantly higher in patients with documented GERD, but it occurred in almost a third of patients without GERD (70 vs. $29 \%, p<0.001$ ).

\section{Prevalence}

Over the past 2 years, several large adult series from the USA and Europe have corroborated $35-43 \%$ of patients with suspected EoE that are eventually diagnosed with PPI-REE $[8,9,10 \bullet \bullet$. As such, a PPI trial before arriving at a diagnosis of EoE is currently mandatory in order to avoid unnecessary therapeutic interventions in PPI-REE, such as topical steroids or elimination diets. Diagnostic and therapeutic implications of PPI-REE have been also included and highlighted in the recently published third (2013) and fourth (2014) iteration of clinical guidelines [11, 12]. Table 1 summarizes the rapidly evolving consideration of PPI responsiveness in patients with suspected EoE over the past 7 years.

\section{Distinguishing features between PPI-REE and EoE}

At the present time, no study has been able to demonstrate the existence of distinguishing phenotypical features between PPI-REE and EoE. Four studies have failed to find distinguishing clinical, endoscopic, and histological features at baseline between patients ultimately found to have EoE or PPI-REE after a PPI trial $[4 \bullet \bullet, 9,10 \bullet \bullet, 13]$. Several studies have also confirmed the lack of useful-

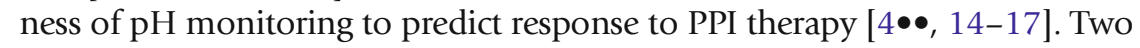
recent studies failed to differentiate between both groups using markers of eosinophilic infiltration and associated inflammatory cells (major basic protein, eotaxin-3, and tryptase) [18] and gene expression of Th2 chemokine (eotaxin-3) and cytokines (interleukin (IL)-5 and IL-13) [10••] in esophageal biopsies.

One of the critical findings in understanding EoE pathogenesis was the discovery of the whole-genome mRNA esophageal expression profile ("EoE

Table 1. Evolving consideration of PPI responsiveness in patients with esophageal eosinophilia compatible with EoE over the past 7 years

\section{7 \\ Consensus Guidelines \\ PPI responsiveness is equivalent to GERD \\ A pathological $\mathrm{pH}$ monitoring is equivalent to either response to PPI therapy or GERD \\ GERD, as an acid-related disorder, is the only showing response to the acid-suppressing ability of PPI therapy}

2011

Consensus Guidelines

PPI responsiveness neither confirms GERD nor excludes EoE

PPI responsiveness might occur either with normal or pathological $\mathrm{pH}$ monitoring

PPI-REE is a new phenotype referring to patients with suspected EoE achieving complete remission on PPI therapy

\section{5}

PPI-REE might be a subphenotype of EoE

PPI-REE and EoE share both genotypical and phenotypical features

PPI therapy in PPI-REE downregulates allergic inflammation in a similar way to steroids in EoE

EOE eosinophilic esophagitis, GERD gastroesophageal reflux disease, PPI proton pump inhibitor, PPI-REE proton pump inhibitor-responsive esophageal eosinophilia 
transcriptome") [19]. More recently, a 94-gene molecular panel, termed as the EoE Diagnostic Panel (EDP), has shown $96 \%$ sensitivity and $98 \%$ specificity to distinguish patients with EoE in remission from controls, as well as identifying patients exposed to glucocorticoids and likely to have relapse after treatment [20]. As such, it would be interesting to ascertain whether this panel can distinguish between PPI-REE and EoE patients. A recent study has been the first to evaluate differences in EDP between untreated 28 EoE and 36 PPI-REE


downregulation pattern comprising the EoE hallmark gene signature was also present in PPI-REE samples at baseline, including increased CCL26 levels for eosinophil chemotaxis, CPA3 levels for mastocytosis, IL-13 responding MUC4 levels, and POSTN levels for tissue remodeling, but not in GERD and control patients. In addition, molecular signature and mastocytosis of untreated PPIREE were almost reversible by using PPI monotherapy in concordance with a previous recent study demonstrating that PPI therapy in PPI-REE downregulated eotaxin- 3 and Th2 cytokines gene expression in esophageal tissue, simi-

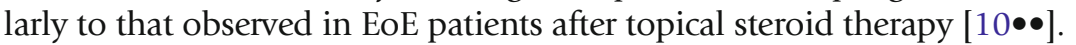

Updated similarities and differences between GERD, PPI-REE and EoE are summarized in Table 2.

\section{PPI-responsive esophageal eosinophilia or PPI-responsive EoE?}

Evolving evidence now shows EoE and PPI-REE are genetically and phenotypically indistinguishable; therefore, questioning whether PPI-REE and EoE are two different diseases [22]. It may be counterintuitive to define PPI-REE by its response to PPI therapy rather than by its genetic, phenotypical, and mechanistic characteristics, which are similar to those of classic EoE. We do not define

Table 2. Updated similarities and differences between GERD, PPI-REE, and EoE

\begin{tabular}{|c|c|c|c|}
\hline & GERD & PPI-REE & EoE \\
\hline Etiology & Gastric content reflux & Unknown & Food/airborne allergens \\
\hline $\begin{array}{l}\text { EoE genetic diagnostic } \\
\text { panel }\end{array}$ & Not expressed & Overexpressed & Overexpressed \\
\hline Symptoms & $\begin{array}{l}\text { Heartburn, regurgitation, } \\
\text { dysphagia }\end{array}$ & $\begin{array}{l}\text { Dysphagia, food bolus } \\
\text { impaction }\end{array}$ & $\begin{array}{l}\text { Dysphagia, food bolus } \\
\text { impaction }\end{array}$ \\
\hline Esophageal involvement & Distal & Distal and proximal & Distal and proximal \\
\hline pH esophageal monitoring & $\begin{array}{l}80 \% \text { increased acid } \\
\text { exposure in erosive GERD } \\
50 \% \text { normal acid exposure } \\
\text { in non erosive GERD }\end{array}$ & $\begin{array}{l}70 \% \text { increased } / 30 \% \\
\text { normal acid exposure }\end{array}$ & $\begin{array}{l}60 \% \text { normal/40 \% } \\
\text { increased acid exposure }\end{array}$ \\
\hline Type of immune & Th1 & Th2 & Th2 \\
\hline $\begin{array}{l}\text { response/involved } \\
\text { chemo/cytokines }\end{array}$ & IL-8, MCP-1, RANTES & Eotaxin-3, IL-13, IL-5 & Eotaxin-3, IL-13, IL-5 \\
\hline Inflammatory cells & $\begin{array}{c}\text { Neutrophils, lymphocytes, } \\
\text { low-grade eosinophilia }\end{array}$ & Eosinophils and mast cells & Eosinophils and mast cells \\
\hline Treatment & $\begin{array}{l}\text { PPI therapy fundoplication } \\
\text { surgery }\end{array}$ & PPI therapy & Steroids/diet \\
\hline
\end{tabular}


bronchial eosinophilic inflammation responsive to corticosteroids as a different entity than asthma when step-up therapy is needed. As such, it is tempting to approach EoE as a disease in which PPI therapy might be the first step in treatment and diet and steroids represent step-up therapy. Subsequently, there is a concern that the term PPI-REE might be replaced by PPI-responsive EoE.

\section{Advances in understanding PPI-REE pathophysiology}

The pathogenesis of esophageal eosinophilia in PPI-REE patients remains unknown. Furthermore, the mechanisms as to why among patients with an identical baseline genotypic and phenotypical expression, some respond to PPI therapy (PPI-REE) while others do not (EoE), are yet to be elucidated. The most well-studied effect of PPIs has been the targeted inhibition of the gastric $\mathrm{H}+\mathrm{K}+-$ ATPase in the parietal cells responsible for gastric acid secretion. Active parietal cells develop an acidic environment wherein PPIs are acid-activated. The active form of the drug then forms covalent disulfide bonds with cysteine residues on the ATPase, rendering the pumps inactive [23].

The most prevailing hypothesis to explain PPI-REE has been assuming GERD to be the priming event. Acid peptic damage to the tight junctions between epithelial cells results in increased permeability with dilation of intercellular spaces, possibly allowing the mucosal penetration of allergens that cause EoE allowing the potential entry of food-derived allergenic molecules through acid-induced epithelial barrier damage [24]. Thus, GERD-induced epithelial damage could expose the deeper layers of the esophageal squamous epithelium to antigens that ordinarily could not penetrate a normal mucosa, facilitating recognition of antigens by antigen-presenting cells to trigger a Th2

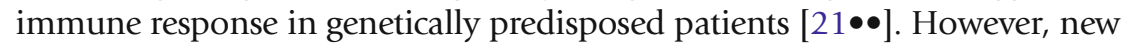
and emerging data suggest that PPIs may benefit patients with EoE through effects that are independent of gastric acid suppression [25•]. A summary of the interplay between potential pathophysiological mechanisms and therapeutic effects of PPI therapy in PPI-REE is displayed in Fig. 1.

\section{Restoration of epithelial barrier function of the esophageal mucosa}

The first study objectively addressing this issue was published in 2014 [26••]. Sixteen patients with esophageal eosinophilia $>15$ eos/HPF were compared to 11 controls at baseline. Esophageal mucosal integrity was measured in the distal esophagus in vivo with a through-the-scope probe during endoscopy and in vitro with two biopsies for electron microscopic analysis of dilated intercellular spaces and four biopsies for measuring transepithelial electrical resistance and transmucosal flux of fluorescently labelled molecules sized 0.3 and $40 \mathrm{kDa}$ (similar to size of food allergens) in Ussing chambers. In patients with esophageal eosinophilia, all measurements of mucosal integrity were significantly impaired when compared to controls. After PPI therapy, half of patients were categorized as PPI-REE. PPI therapy resulted in partial restoration of mucosal integrity in PPI-REE, but not in EoE patients. The authors concluded that mucosal integrity impairment in PPI-REE might be due to GERD, whereas it might be related to inflammatory cell recruitment in EoE. However, these results should be viewed with caution since acid reflux was not measured by $\mathrm{pH}$ monitoring and barrier healing related to potential anti-inflammatory effects of PPI therapy could not be positively ruled out. 
EoE patients have been recently shown to have lower thresholds for onset of symptoms and pain after esophageal acid infusion when compared to healthy volunteers [27]. As a matter of fact, EoE patients without GERD showed an earlier burning sensation than EoE patients with concomitant GERD or healthy volunteers after esophageal acid infusion [27]. Suppression of gastric acid secretion with PPIs, thus, might provide symptomatic relief even for patients with either normal or pathological esophageal acid exposure. This phenomenon, therefore, might explain why several series of EoE patients have been reported to achieve complete symptom remission on PPI therapy, despite persistent esophageal inflammation $[4 \bullet \bullet, 10 \bullet \bullet, 16,28-31]$. Furthermore, a significant reduction in distal esophageal eosinophil load (not reaching the

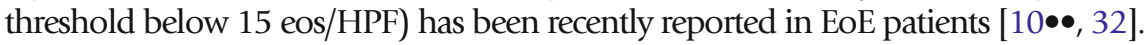

\section{Anti-inflammatory effects of PPI therapy}

Esophageal acid exposure in GERD can enhance the expression of adhesion molecules (e.g., vascular cell adhesion protein-1) and Th1 cytokines (e.g., IL-8 and IL-1b) and cause the release of other inflammatory mediators in the esophagus, leading to the recruitment of neutrophils, lymphocytes, and, to a lesser extent, eosinophils. In 2009, a provocative experimental study demonstrated that GERD, in contrast to classic thinking, caused esophageal inflammation through a cytokine-mediated mechanism rather than direct epithelial caustic injury [33]. In this study, the authors observed that after surgical induction of reflux, the first histologic inflammatory response detected was a lymphocytic infiltration of the submucosa which progressed to the mucosal surface. Interestingly, mucosal erosions did not appear until postoperative week 4. These findings suggest that reflux esophagitis develops primarily as an immune-related injury rather than solely as a caustic chemical injury. As such, one can speculate that in atopic patients, GERD may not induce a Th1 cytokine injury through a pathway similar to the Th2 pathway found in EoE. In this regard, a recent experimental study has shown that eotaxin-3 expression in GERD and EoE cell cultures is similar when stimulated with Th2 cytokines [34••], posing the possibility that in patients at risk for EoE, such as those with other atopic disorders, the injury of GERD may be diverted to an alternate pathway from typical erosive to EoE. More data are needed to fully assess this pathway.

Interestingly, new and emerging data suggest that PPIs may benefit patients with PPI-REE and EoE through effects that are independent of gastric acid suppression. Two recent experimental studies $[34 \bullet \bullet, 35]$ have shown that PPIs inhibit Th2 cytokine-stimulated secretion of eotaxin-3, the primary eosinophil chemoattractant in EoE, in esophageal squamous epithelial cells. Both omeprazole and lansoprazole exhibited this inhibition, suggesting a class effect of PPIs. This inhibition occurred with omeprazole concentrations as low as $1 \mathrm{mmol} / \mathrm{l}$, which are physiologically achievable in blood with conventional oral dosing or intravenous administration. Mechanistically, the inhibitory effect of PPIs seems to involve chromatin remodeling in the eotaxin-3 promoter, resulting in decreased promoter binding of the transcription factor protein, signal transducer, and activator of transcription (STAT)6, decreased RNA polymerase II recruitment, and, overall, reduced eotaxin-3 transcriptional activity in 
esophageal squamous epithelial cells [35]. As mentioned above, these potential anti-inflammatory effects have been recently corroborated in clinical studies $[10 \bullet \bullet, 21 \bullet \bullet]$.

Future perspectives in PPI-REE

Multiple diagnostic and therapeutic dilemmas surrounding PPI-REE are shown in Table 3. The precise etiology and mechanistic pathophysiology of PPI-REE remain unknown (Fig. 1). Another main issue is we do not understand why among identical patients, some respond to PPIs (PPI-REE) yet others do not (EoE). Following the lead of other diseases, there may be genomic differences or subtle variations in disease pathways that explain therapeutic response. In the first study evaluating the EoE diagnostic panel in PPI-REE and EoE patients, KCNJ2 (potassium inwardly rectifying channel, subfamily J, member 2/Kir2.1) became the only gene with significant differential expression between both disorders $[21 \bullet \bullet]$ KCNJ2 resulted in $72 \%$ sensitivity and $72 \%$ specificity to predict PPI-REE-pre versus EoE. KCNJ2 encodes the potassium channel Kir2.1, which is abundant in gastrointestinal mucosa and colocalizes with the H1-K1ATPase/proton pump. Therefore, the authors propose a potential interaction between this potassium channel and proton pump in the upper gastrointestinal epithelium to explain PPI-REE.

Another important unsolved issue is to ascertain whether PPI-REE and EoE also share a similar natural history and long-term management. EoE has been demonstrated to be a chronic disease with persistence of symptoms and inflammation over years [36]. Furthermore, long-standing eosinophilic inflammation may increase the risk of esophageal remodeling with subsequent stricture formation. A recent study nicely showed how the prevalence of esophageal strictures correlates with the duration of untreated disease [37]. Eosinophils, eotaxin-3, and Th2 cytokines (IL-5 and IL-13) have been shown to be necessary for this esophageal tissue remodeling in EoE. In addition, swallowed topical corticosteroids and dietary interventions not only reduce this inflammation but may also prevent and even reverse this esophageal remodeling process in the long run [38]. Given the fact that PPI-REE and EoE share genotypic, phenotypical, and mechanistic features, there is concern that PPI-REE and EoE might share a similar risk of fibrostenotic complications if untreated, and hopefully, PPI therapy might impact on this natural history in PPI-REE. Two small retrospectives series of patients have shown that children with PPI-REE may

\section{Table 3. Unsolved issues regarding diagnosis and treatment for PPI-REE}

Etiology and pathogenesis of PPI-REE

Molecular biomarkers/genetic testing distinguishing PPI-REE from EoE

Natural history of PPI-REE

Doses, dosing interval, and duration required for initial PPI trial

Influence of PPI metabolism genotype (CYP2C19) in short- and long-term PPI response

Optimal PPI dosing as maintenance therapy

Rate of sustained remission on maintenance PPI therapy

Impact of allergen exposure on sustained PPI responsiveness

Need and frequency of endoscopy in follow-up

EOE eosinophilic esophagitis, PPI proton pump inhibitor, PPI-REE proton pump inhibitor-responsive esophageal eosinophilia 
eventually progress to EoE [39, 40]. A recent abstract has first evaluated longterm response to PPI therapy in PPI-REE [41]. In 40 PPI-REE patients, acid suppressive therapy was progressively tapered based on clinical symptoms and maintained at the lowest dose with the target endpoint of clinical remission, with a follow-up endoscopy performed at 12 months or longer. Sustained clinico-histological remission on low-dose maintenance PPI therapy was observed in $64 \%$ of adult PPI-REE patients. A majority of relapsers showed recurrent eosinophilic inflammation limited to the distal esophagus, which resolved after PPI dose intensification. Therefore, it might be uncommon for adult PPI-REE patients to fully lose PPI response and be reclassified as EoE.

Current guidelines $[5 \bullet, 11,12]$ suggest high-dose PPI therapy (20-40 mg once or twice daily in adults and $1 \mathrm{mg} / \mathrm{kg}$ per dose twice daily in children for 812 weeks). However, we lack comparative studies in terms of PPI molecule, doses, dosing interval and duration. PPIs undergo hepatic metabolism by the cytochrome P450 (CYP) system and the principal enzyme involved in the activation process of PPIs is CYP2C19. This genotype influences response to PPI therapy in PPI-based therapies, such as GERD or eradication therapy for Helicobacter pylori infection [42, 43]. A rapid metabolizer genotype, which is the most common genotype in Caucasian populations (56-81\%), may result in low plasma levels and lack of therapeutic effect of PPI therapy in PP-REE patients, both in the short and long term. Further studies should validate this hypothesis.

\section{Compliance with Ethics Guidelines}

\section{Conflict of Interest}

Javier Molina-Infante declares that he has no conflict of interest.

Alfredo J. Lucendo declares that he has no conflict of interest.

\section{Human and Animal Rights and Informed Consent}

This article does not contain any studies with human or animal subjects performed by the authors.

\section{References and Recommended Reading}

Papers of particular interest, published recently, have been highlighted as:

- Of importance

$\bullet$ Of major importance

1. Hruz P, Straumann A, Bussmann C, Heer P, Simon HU, Zwahlen $\mathrm{M}$, et al. Escalating incidence of eosinophilic esophagitis: a 20-year prospective, population-based study in Olten County, Switzerland. J Allergy Clin Immunol. 2011;128:1349-50.

2. Furuta GT, Liacouras CA, Collins MH, Gupta SK, Justinich C, Putnam PE, et al. Eosinophilic esophagitis in children and adults: a systematic review and consensus recommendations for diagnosis and treatment. Gastroenterology. 2007;133:1342-63.
3. Ngo P, Furuta GT, Antonioli DA, Fox VL. Eosinophils in the esophagus: peptic or allergic eosinophilic esophagitis? Case series of three patients with esophageal eosinophilia. Am J Gastroenterol. 2006;101:1666-70.

4.• Molina-Infante J, Ferrando-Lamana A, Ripoll C, Hernandez-Alonso M, Mateos JM, Fernandez Bermejo $\mathrm{M}$, et al. Esophageal eosinophilic infiltration responds to proton pump inhibition in most adults. Clin Gastroenterol Hepatol. 2011;9:110-7. 
This is the first prospective study highlighting the high prevalence of PPI-REE in patients with suspected EoE, questioning also the role of $\mathrm{pH}$ monitoring for predicting responsiveness to PPI therapy and to definitely rule out EoE.

5. $\quad$ Liacouras CA, Furuta GT, Hirano I, Atkins D, Attwood SE, Bonis PA, et al. Eosinophilic esophagitis: updated consensus recommendations for children and adults. J Allerg Clin Immunol. 2011;128:3-10.

This is the first guideline specifically addressing PPI-REE and EoE as potential distinct entities. PPI-REE was suggested not to be necessarily a manifestation of GERD, since it might occur with pathological or normal acid esophageal exposure.

6. Spechler SJ, Genta RM, Souza RF. Thoughts on the complex relationship between gastroesophageal reflux disease and eosinophilic esophagitis. Am J

Gastroenterol. 2007;102:1301-6.

7. Molina Infante J, Katzka DA, Gisbert JP. Review article: proton pump inhibitor therapy for suspected eosinophilic esophagitis. Aliment Pharmacol Ther. 2013;37:1157-64.

This is the first systematic review on PPI-REE in children and adults. Ten articles comprising 258 patients with suspected EoE (152 children, 106 adults) revealed that at least one third of patients with were actually PPI-REE patients. Histological remission after PPI therapy was reported higher in adults than in children (23-40\% vs. 33-61\%) and in patients with documented GERD when compared to those with no GERD on endoscopy/pH monitoring (70\% vs. 29\%, $p<0.001$ ).

8. Vazquez-Elizondo G, Ngamruengphong S, Khrisna M, Devault KR, Talley NJ, Achem SR. The outcome of patients with oesophageal eosinophilic infiltration after an eight-week trial of a proton pump inhibitor. Aliment Pharmacol Ther. 2013;38:1312-9.

9. Dellon ES, Speck O, Woodward K, Covey S, Rusin S, Gebhart JH, et al. Clinical and endoscopic characteristics do not reliably differentiate PPI-responsive esophageal eosinophilia and eosinophilic esophagitis in patients undergoing upper endoscopy: a prospective cohort study. Am J Gastroenterol. 2013;108:1854-60.

$10 . \bullet$ Molina-Infante J, Rivas MD, Hernandez-Alonso M, Vinagre-Rodriguez G, Mateos Rodriguez JM, DueñasSadornil C, et al. Remission in proton pump inhibitorresponsive esophageal eosinophilia correlates with downregulation of eotaxin-3 and TH2 cytokines, similarly to eosinophilic esophagitis after steroids. Aliment Pharmacol Ther. 2014;40:955-65.

This is the first study demonstrating PPI therapy downregulates eotaxin- 3 and Th2 cytokines in PPI-REE patients, in a similar way to than seen with topical steroids in EoE patients. This study represents the first in vivo demonstration of potential anti-inflammatory effects of PPI therapy.

11. Dellon ES, Gonsalves N, Hirano I, Furuta GT, Liacouras CA, Kaztka DA. ACG clinical guideline: evidenced based approach to the diagnosis and management of esophageal eosinophilia and eosinophilic esophagitis (EoE). Am J Gastroenterol. 2013;108:679-92.

12. Papadopoulou A, Koletzko S, Heuschkel R, Dias JA, Allen KJ, Murch SH, et al. Management guidelines of eosinophilic esophagitis in childhood. J Pediatr Gastroenterol Nutr. 2014;58:107-18.

13. Moawad FJ, Schoepfer AM, Safroneeva E, Ally MR, Chen YJ, Maydonovitch CL, et al. Eosinophilic oesophagitis and proton pump inhibitor-responsive oesophageal eosinophilia have similar clinical, endoscopic and histological findings. Aliment Pharmacol Ther. 2014;39:603-8.

14. Dranove JE, Horn DS, Davis MA, Kernek KM, Gupta SK. Predictors of response to proton pump inhibitor therapy among children with significant esophageal eosinophilia. J Pediatr. 2009;154:96-100.

15. Peterson KA, Thomas KL, Hilden K, Emerson LL, Wills JC, Fang JC. Comparison of esomeprazole to aerosolized, swallowed fluticasone for eosinophilic esophagitis. Dig Dis Sci. 2010;55:1313-9.

16. Francis DL, Foxx-Orenstein A, Arora AS, Smyrck TC, Jensen K, Nord SL, et al. Results of ambulatory pH monitoring do not reliably predict response to therapy in patients with eosinophilic esophagitis. Aliment Pharmacol Ther. 2012;35:300-7.

17. Moawad FJ, Veerappan GR, Dias JA, Baker TP, Maydonovitch CL, Wong RK. Randomized controlled trial comparing aerosolized swallowed fluticasone to esomeprazole for esophageal eosinophilia. Am J Gastroenterol. 2013;108:366-72.

18. Dellon ES, Speck O, Woodward K, Covey S, Rusin S, Gebhart JH, et al. Markers of eosinophilic inflammation for diagnosis of eosinophilic esophagitis and proton pump inhibitor-responsive esophageal eosinophilia: a prospective study. Clin Gastroenterol Hepatol. 2014;25(12):2015-22.

19. Blanchard C, Wang N, Stringer KF, Mishra A, Fulkerson PC, Abonia JP, et al. Eotaxin-3 and a uniquely conserved gene-expression profile in eosinophilic esophagitis. J Clin Invest. 2006;116:536-47.

20. Wen T, Stucke EM, Grotjan TM, Kemme KA, Abonia JP, Putnam PE, et al. Molecular diagnosis of eosinophilic esophagitis by gene expression profiling. Gastroenterology. 2013;145:1289-99.

21.•Wen T, Dellon ES, Moawad FJ, Furuta GT, Aceves SS, Rothenberg ME. Transcriptome analysis of proton pump inhibitor-responsive esophageal eosinophilia reveals proton pump inhibitor-reversible allergic inflammation. J Allergy Clin Immunol. 2015;135(1):187-97.

This is the first study evaluating EoE transcriptome in PPI-REE patients, which was basically indistinguishable from that of EoE patients, but different from that of GERD and control patients. These results support PPI-REE being a subphenotype of EoE rather than a distinct entity. The EoE transcriptome was almost reversible with PPI monotherapy.

22. Katzka DA. Eosinophilic esophagitis and proton pump-responsive esophageal eosinophilia: what is in a name? Clin Gastroenterol Hepatol. 2014;12:2023-5.

23. Schubert ML, Peura DA. Control of gastric acid secretion in health and disease. Gastroenterology. 2008; 134:1842-60. 
24. Tobey NA, Hosseini SS, Argote CM, et al. Dilated intercellular spaces and shunt permeability in nonerosive acid-damaged esophageal epithelium. Am J

Gastroenterol. 2004;99:13-22.

25.• Molina-Infante J, Katzka DA, Dellon ES. Proton pump inhibitor-responsive esophageal eosinophilia: a historical perspective on a novel and evolving entity. Rev Esp Enf Dig. 2015;107:29-36.

A historical review on how diagnostic considerations for esophageal eosinophilia and response to PPI therapy have changed enormously over the last for decades, and especially since 2007.

26.• van Rhijn BD, Weijenborg PW, Verheij J, van der Bergh Weerman MA, Verseijden C, van den Wijngaard RM, et al. Proton pump inhibitors partially restore mucosal integrity in patients with proton pump inhibitor-responsive esophageal eosinophilia but not eosinophilic esophagitis. Clin Gastroenterol Hepatol. 2014;12:1815-23.

This is the first study in which the esophageal mucosal integrity has been assessed in PPI-REE and EoE. PPI therapy partially restored mucosal integrity in PPI-REE, but not in EoE patients, suggesting that increased permeability of the esophageal mucosa may play a role in the pathogenesis of PPI-REE.

27. Krarup AL, Villadsen GE, Mejlgaard E, et al. Acid hypersensitivity in patients with eosinophilic oesophagitis. Scand J Gastroenterol. 2010;45:273-81.

28. Desai TK, Stecevic V, Chang CH, et al. Association of eosinophilic inflammation with esophageal food impaction in adults. Gastrointest Endosc. 2005;61:795801.

29. Sayej WN, Patel R, Baker RD, et al. Treatment with high-dose proton pump inhibitors helps distinguish eosinophilic esophagitis from noneosinophilic esophagitis. J Pediatr Gastroenterol Nutr. 2009;49:1-7.

30. Abe Y, Iijima K, Ohara S, et al. A Japanese case series of 12 patients with esophageal eosinophilia. J Gastroenterol. 2011;46:25-30.

31. Levine J, Lai J, Edelman M, Schuval SJ. Conservative long-term treatment of children with eosinophilic esophagitis. Ann Allergy Asthma Immunol. 2012;108:363-6.

32. Straumann A, Hoesli S, Bussmann C, et al. Antieosinophil activity and clinical efficacy of the CRTH2 antagonist OC000459 in eosinophilic esophagitis. Allergy. 2013;68:375-85.

33. Souza RF, Huo X, Vittal M, Schuler CM, Carmack SW, Zhang HY, et al. Gastroesophageal reflux might cause esophagitis through a cytokine-mediated mechanism rather than caustic acid injury. Gastroenterology. 2009;137:1776-84.
34.• Cheng E, Zhang X, Huo X, Yu C, Zhang Q, Wang DH, et al. Omeprazole blocks eotaxin-3 expression by oesophageal squamous cells from patients with eosinophilic oesophagitis and GORD. Gut. 2013;62:824-32. This is the first experimental study showing that GERD and EoE cells share the potential to express eotaxin-3 whenever stimulated by Th2 cytokines. Moreover, omeprazole was shown to block eotaxin-3 expression in both GERD and EoE patients. As such, PPI might have anti-inflammatory effects independent of acid suppression and a PPI response may not distinguish accurately between GERD and EoE.

35. Zhang X, Cheng E, Huo X, Yu C, Zhang Q, Pham TH, et al. Omeprazole blocks STAT6 binding to the eotaxin3 promoter in eosinophilic esophagitis cells. PLoS One. 2012;7:e50037.

36. Straumann A, Spichtin HP, Grize L, Bucker KA, Beglinger C, Simon HU. Natural history of primary eosinophilic esophagitis: a follow-up of 30 adult patients for up to 11.5 years. Gastroenterology. 2003;125:1660-9.

37. Schoepfer AM, Safroneeva E, Bussmann C, Kuchen T, Portmann S, Simon HU, et al. Delay in diagnosis of eosinophilic esophagitis increases risk for stricture formation in a time-dependent manner. Gastroenterology. 2013;145:1230-6.

38. Straumann A, Schoepfer A. Update on basic and clinical aspect of eosinophilic oesophagitis. Gut. 2014;63:1355-63.

39. Dohil R, Newbury RO, Aceves S. Transient PPI responsive esophageal eosinophilia may be a clinical sub-phenotype of pediatric eosinophilic esophagitis. Dig Dis Sci. 2012;57:1413-9.

40. Schroeder S, Capocelli KE, Masterson JC, Harris R, Protheroe C, Lee JJ, et al. Effect of proton pump inhibitor on esophageal eosinophilia. J Pediatr Gastroenterol Nutr. 2013;56:166-72.

41. Molina-Infante J, Martinek J, Martinez-Alcala C, Krajciova J, Moawad FJ, Dellon ES. Long-term efficacy of PPI therapy in patients with PPI-responsive esophageal eosinophilia: an international multicenter study. Gastroenterology. 2014;146 Suppl 1:S-393.

42. Furuta T, Shirai N, Sugimoto $M$, et al. Influence of CYP2C19 pharmacogenetic polymorphism on proton pump inhibitor-based therapies. Drug Metab Pharmacokinet. 2005;20:153-67.

43. Tang HL, Li Y, Hu YF, Xie HG, Zhai SD. Effects of CYP2C19 loss-of function variants on the eradication of $H$. pylori infection in patients treated with proton pump inhibitor-based triple therapy regimens: a meta analysis of randomized clinical trials. PLoS One. 2013;8:e62162. 